\title{
Design of Perturbation Signals for the Estimation of Proprioceptive Reflexes
}

\author{
Alfred C. Schouten*, Erwin de Vlugt, and Frans C. T. van der Helm
}

\begin{abstract}
This study aimed to identify the functional contribution of reflexes to human motor control during posture maintenance. Continuous random force disturbances were applied at the hand while the subjects were instructed to minimize the deviation resulting from the force disturbances. The results were analyzed in the frequency domain with frequency response functions (FRFs). Two FRFs were evaluated: 1) the mechanical admittance and 2) the reflexive impedance, expressing the dynamic relation between position and muscle activation (assessed via electromyography, EMG). The reflexive impedance is a direct measure of the proprioceptive reflexes. To record all relevant dynamical characteristics of the arm, wide bandwidth signals were used as force disturbance. Distributing the power of the signal over fewer frequencies within the bandwidth improved the signal-to-noise-ratio SNR of the EMG recordings, facilitating reliable estimation of the reflexive impedance. The coherence indicated that the relation between force disturbance and EMG is linear under the given conditions and improved with the SNR. The method of designing disturbance signals and the estimation of the reflexive impedance are useful for studies aiming to quantify proprioceptive reflexes and to investigate its functionality.
\end{abstract}

Index Terms-Arm admittance, electromyography (EMG), identification, proprioceptive reflexes, reflexive impedance.

\section{INTRODUCTION}

$\mathbf{T}$ HE DYNAMICS of a human joint comprise inertial and visco-elastic properties of (active) muscles and passive tissues as well as proprioceptive reflexes from afferent feedback. Several studies showed that the strength of reflexive feedback varies with task and conditions [1]-[8].

Previous studies around the ankle applied continuous random position perturbations to estimate the relation from ankle angle to torque and surface electromyography (EMG) [4], [5]. These studies found that the stretch reflex is dominated by a velocity sensitivity in series with a neural time delay. The sensitivity of the stretch reflex increased with tonic activation level of the muscle and decreased with displacement amplitude. A succeeding study [6] investigated the contribution of the stretch reflex to the joint dynamics. However, as the EMG was expressed in arbitrary units, assumptions were needed to separate the reflexive and active muscle contributions. It was found that the reflex mechanisms were most important in the $5-10 \mathrm{~Hz}$ range and

Manuscript received October 25, 2006; revised October 8, 2007. Asterisk indicates corresponding author.

*A. C. Schouten is with the Department of Biomechanical Engineering, Delft University of Technology, 2628 CD Delft, The Netherlands (e-mail: a.c.schouten@tudelft.nl).

E. de Vlugt and F. C. T. van der Helm are with the Department of Biomechanical Engineering, Delft University of Technology, 2628 CD Delft, The Netherlands (e-mail: e.devlugt@tudelft.nl; f.c.t.vanderhelm@tudelft.nl).

Digital Object Identifier 10.1109/TBME.2007.912432 that the reflex torque magnitude was in the same magnitude as intrinsic mechanisms.

Continuous random force disturbances proved to be useful to quantify the functional contribution of reflexes around the shoulder during active tasks [7]-[9]. Force disturbances with position tasks (minimize displacements) are unambiguous and natural for the subject: the subject must actively control the position and preserve stability. These studies showed that the reflexive contribution varied with the frequency content of the force disturbance and the external dynamics applied by the manipulator. These studies confirmed that like the ankle also for the shoulder, the reflex and intrinsic contributions were in the same order of magnitude. Model simulation showed that the reflex adaptations were optimal to minimize the displacements for the given conditions [7], [10], [11]. However, these studies were all based on analyzes of the dynamic relation between force and position (mechanical admittance) only, and consequently, assumptions were needed to separate the contributions. Incorporation of the EMG in the procedures, as a measure for muscle activation, might improve the procedures.

High effort tasks like "minimize displacements" [7]-[9] demand high levels of cocontraction. This implies that the variation in the muscle activation due to reflexes will be relatively small as compared to the high tonic muscle activity. Muscle activity is easily recorded by the EMG. The EMG, however, contains substantial noise, and especially with high tonic activations, the small variations due to reflexes are hard to identify. The goal of this paper is to redesign the continuous signals used as force disturbances such that the dynamic relation between position and EMG can be estimated reliably in addition to the mechanical admittance during active postural control tasks.

In this paper, the shoulder joint was investigated and disturbances were applied in a single degree-of-freedom. It is desired to estimate the functional contribution of reflexes to the total mechanical joint admittance. As multiple muscles contributed to the torque, a lumped muscle activation was constructed based on the EMGs of the main shoulder muscles. By scaling the EMGs to muscle force, the net (lumped) muscle torque could be estimated. The reflexive impedance was introduced and defined as the dynamic relation between displacement and lumped muscle activation, where the latter was scaled in force.

This study demonstrated that by a proper signal design, it was possible to reliably estimate the frequency response functions (FRFs) for the reflexive impedance and mechanical admittance around the shoulder. The high coherence, especially for frequencies above $1.5 \mathrm{~Hz}$, showed that use of (linear) FRFs was justified for the given conditions: small displacement around a working point. The reflex system itself is clearly nonlinear, as the 


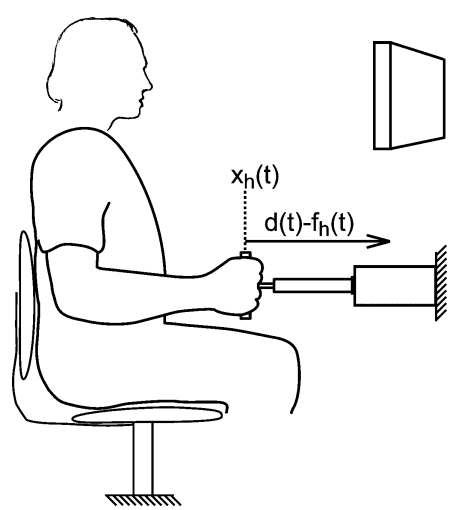

Fig. 1. Experimental setup; the subject sits on a chair and holds the handle with the right hand. The piston can move forward and backward. The hand force, $f_{h}(t)$, applied to the hydraulic manipulator is measured by a force transducer mounted between the handle and the piston. The manipulator controls the position of the handle, $x_{h}(t)$, and is based on the hand force, the external force disturbance, $d(t)$, and the simulated external dynamics (or environment).

magnitude varies with task and conditions. A succeeding study will use the signal design procedure to investigate the effect of disturbance bandwidth and environment on proprioceptive reflexes, by fitting a model on the mechanical admittance and reflexive impedance simultaneously [12].

\section{MATERIAL AND METHODS}

\section{A. Subjects}

Ten healthy subjects participated in the experiment (20-44 years, four women, three left handed). The experiments were conducted on the right arm only. All subjects gave informed consent prior to the experiment.

\section{B. Apparatus}

A manipulator was used to apply force disturbances to the shoulder joint, see Fig. 1. The manipulator was described in detail previously [8], [13]. The subject was seated in a chair while holding the handle of a linear actuator. The subject was positioned in such a way that the elbow made an angle of $90^{\circ}$ of flexion. The subject was able to move the handle of the manipulator for- and backward, resulting in forward rotation of the gleno-humeral joint [14]. The manipulator was forcecontrolled, and interaction between the subject and manipulator existed, i.e., the position of the handle depended on both the dynamics of the subject and the external environment imposed by the manipulator, see Fig. 2. The manipulator "felt" like a mass-spring-damper system to the subject. The parameters of this (virtual) environment were adjustable. In this study, the mass, $m_{e}$, was set to a fixed value of $1 \mathrm{~kg}$. No virtual damper and spring were used $\left(b_{e}=0 \mathrm{Ns} / \mathrm{m}, k_{e}=0 \mathrm{~N} / \mathrm{m}\right)$.

\section{Procedures}

Subjects had to hold the handle and were instructed to minimize the displacements of the handle, while continuous random force disturbances were applied for $30 \mathrm{~s}$. All subjects experienced the task as natural and motivating. Only a few trials were

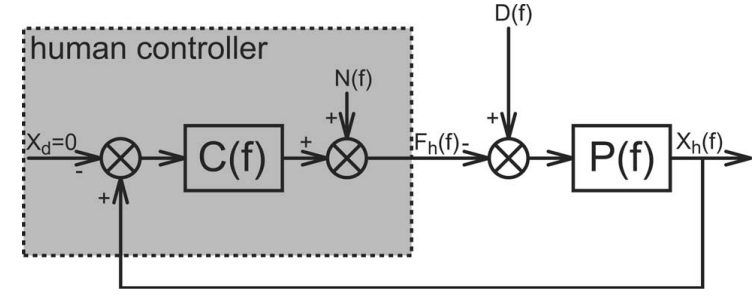

Fig. 2. Blockscheme of human posture control expressed in the frequency domain. $C(f)$ : transfer function of arm dynamics; $P(f)$ : transfer function of manipulator dynamics; $D(f)$ : external force disturbance; $F_{h}(f)$ : hand reaction force; $X_{h}(f)$ : hand position (coincides with the manipulator handle); $N(f)$ : model remnant; $X_{d}=0$ : reference position. The human arm dynamics (gray box) are described by the linear transfer function, $C(f)$, together with remnant $N(f)$, which is uncorrelated with $D(f)$.

necessary to get the subject acquainted with the manipulator. The actual position of the handle was shown on a display to assist and motivate the subjects and to prevent drift.

Six different signals were used as a force disturbance. All signals had a bandwidth of $0.5-20 \mathrm{~Hz}$, which was sufficient to capture all relevant dynamics of the arm [8]. The force disturbances were composed as a sequence of sines, the so-called multisine signals. These signals were designed to have a specified frequency content, and by minimizing the crest factor (the compression, or compactness, of the signal), the power of the signal could be increased without changing signal amplitude [15]-[17]. The crest factor is defined as the maximal amplitude of the signal divided by the root-mean-square (RMS) of the signal:

$$
\mathrm{CF}=\frac{\max |d(t)|}{\mathrm{RMS}_{d}}
$$

in which CF is the crest factor and $\mathrm{RMS}_{d}$ denotes the RMS of signal $d(t)$.

Random signals have crest factors in the range of 4-5, while optimized signals have crest factors around 2. When the amplitude of the output is limited, e.g., the position deviations must be kept small, higher input power can be used with signals having an optimized crest factor. Higher input power generally results in a better SNR, and consequently, will improve the estimators for the FRFs between input and output.

The signals were $30 \mathrm{~s}$ in length and had a sample frequency of $2500 \mathrm{~Hz}$. For analysis, the first 9464 samples $(\approx 4 \mathrm{~s})$ were omitted to remove any initial transient response, leaving $2^{16}$ samples $(\approx 26 \mathrm{~s})$ for analysis. As a result of the limited observation time $\left(T_{\mathrm{obs}}=26.2144 \mathrm{~s} \equiv 2^{16}\right.$ samples $)$, a finite resolution in the frequency domain resulted $\left(\Delta f=T_{\mathrm{obs}}{ }^{-1} \approx 0.038 \mathrm{~Hz}\right.$ ). As a result of the finite resolution in the frequency domain, 508 sines existed in the bandwidth of $0.5-20 \mathrm{~Hz}$. The method used to estimate the FRFs required averaging over four adjacent frequency bands (see Section II-D2), resulting in 127 clusters of four adjacent frequencies within the bandwidth.

In this study, six different multisine signals were tested. The reference signal (WB127) had a full spectrum, i.e., uniform power in all clusters between 0.5 and $20 \mathrm{~Hz}$. To further improve the SNR of the EMG, the number of clusters was gradually reduced from 64 to 22 clusters (WB64, WB43, WB32, WB26, and WB22). In all signals with reduced spectra, the power was uniformly and equidistantly distributed over the signal bandwidth. 

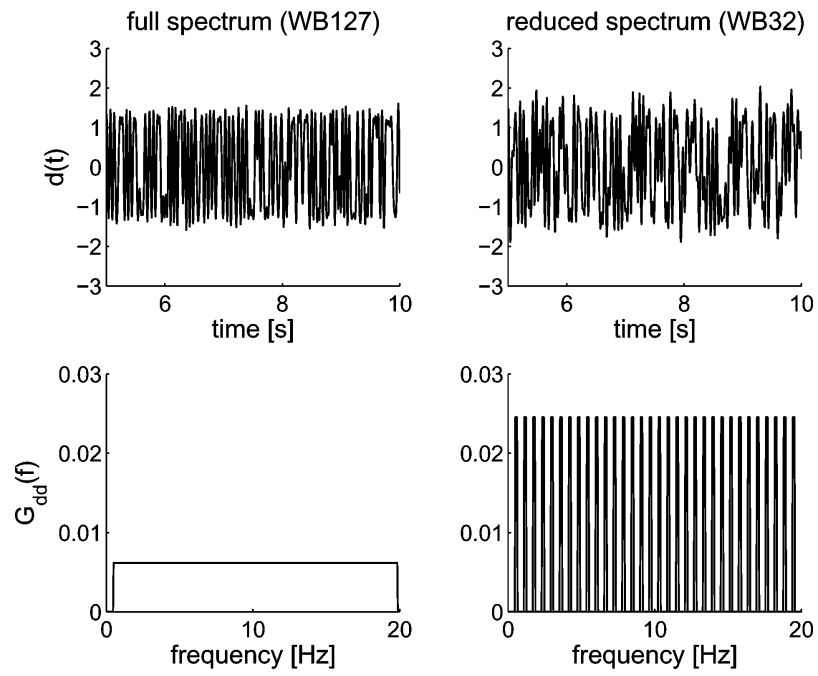

Fig. 3. Left: full spectrum disturbance WB127; right: reduced spectrum disturbance WB32. Upper plots: $5 \mathrm{~s}$ fragment of the signals; lower plots: power spectral density of the signals. Both signals are scaled to have an RMS of 1.

In Fig. 3, the full spectrum signal and a reduced spectrum signal are shown. The power spectral densities indicate the distribution of signal power over the frequencies. Because in the reduced spectrum signals the power is distributed over fewer clusters, the power per frequency increases, while the total power, and consequently, the RMS of the signals were constant.

To obtain adequate linear model approximations, the position deviations must be kept small. Prior to the experiment, the magnitude of the reference force disturbance was adjusted by trial and error to obtain an RMS for the hand position of approximately $3 \mathrm{~mm}$. This signal magnitude was set once and set equal for all disturbance signals. Each force disturbance was applied four times in random order resulting in 24 trials. Subjects indicated when they were ready for the next trial, typically the time between trials was 10-20 s, and most subjects had one longer break of a few minutes.

The EMG amplitude differs for each muscle and each subject as a result of different anatomical and physiological conditions. To relate the EMG to muscle force, the EMG to force ratios were determined from isometric push and pull tasks performed prior and after the experiment. The subjects had to maintain constant force levels for $10 \mathrm{~s}(-25,-20,-15,0,15,20,25 \mathrm{~N})$ by pushing or pulling on the handle, while the handle was controlled to be in a fixed (rigid) position. During the isometric trials, the force at the handle was shown on the display to assist the subject. The EMG to force ratio was determined by linear regression.

\section{Data Processing}

1) Signal recording and processing: During a trial, the force disturbance, $d(t)$, the position of the handle, $x_{h}(t)$, the force at the handle, $f_{h}(t)$, and the EMG signals of four relevant shoulder muscles $\left(e_{1}: \mathrm{m}\right.$. pectoralis major, $e_{2}: \mathrm{m}$. deltoideus anterior, $e_{3}: \mathrm{m}$. deltoideus posterior, and $e_{4}: \mathrm{m}$. latissimus dorsi) were recorded at $2500 \mathrm{~Hz}$ sample frequency and 16-b resolution. Before recording, the EMG signals were high pass filtered to remove dc components and movement artifacts $(20 \mathrm{~Hz}$, thirdorder Butterworth) and low pass filtered to prevent aliasing ( $1 \mathrm{kHz}$, third-order Butterworth). This study investigated stationary behavior, and to remove any initial transient effect, the first 9464 samples $\left(\approx 4\right.$ s) were eliminated, leaving $2^{16}$ samples $(\approx 26 \mathrm{~s})$ for further processing.

The EMG recordings of the muscles were used: 1) to estimate the amount of coactivation during a trial expressed by the mean EMG, $u_{0}$, and 2) to obtain the (lumped) muscle activation, $a(t)$, to get a measure of the active state of the muscles at time $t$.

To improve the quality of the EMG signals, a prewhitening filter was implemented, following the procedures as described in [18]. Recorded bipolar EMG has a colored spectrum primarily resulting from spatial filtering. In prewhitening, an inverse filter was applied to white the recorded EMG, i.e., to restore the flat (white) spectrum. The EMG signals during the maximum isometric push and pull tasks were used to obtain the parameters of the prewhitening filter following the procedures of [18]. First, the power spectral density (PSD) of the EMGs were estimated. Second, the PSDs were described by fitting an autoregressive filter (sixth order) onto the PSD. The prewhitening filter was implemented as a moving average filter, which is the inverse of an autoregressive filter, using the parameters of the fitted autoregressive filter.

The integrated rectified EMG (IEMG) was calculated for each trial

$$
\mathrm{IEMG}_{i}=\frac{1}{n} \sum_{k=1}^{n}\left|e_{w, i}\left(t_{k}\right)\right|
$$

in which $e_{w, i}$ is the prewhitened EMG of muscle $i, t_{k}$ is the indexed time vector, and $n$ is the number of samples. The mean EMG was calculated according to

$$
u_{0}=\frac{1}{4} \sum_{i=1}^{4} \frac{\text { IEMG }_{i}}{\text { IEMG }_{\mathrm{ref}, \mathrm{i}}}
$$

in which IEMG $_{\mathrm{ref}, \mathrm{i}}$ denotes the IEMG for muscle $i$ during the reference disturbance (WB127) averaged over the four repetitions.

To calculate the (lumped) muscle activation, the EMG signals were scaled and expressed in Newtons. For each muscle, the EMG to force ratio, $K_{i}$, was obtained by fitting a straight line between the average forces and the average IEMGs of the isometric push/pull tasks, using a least squares criterion. The ratios of the "push" muscles were determined (m. pectoralis major and $\mathrm{m}$. deltoideus anterior) from the isometric push tasks, and the ratios of the "pull" muscles (m. deltoideus posterior and m. latissimus dorsi) from the pull tasks. The muscle activation was obtained by combining the rectified prewhitened EMGs of the four recorded muscles:

$$
\begin{aligned}
a(t)= & \frac{1}{2}\left(K_{1}\left|e_{w, 1}(t)\right|+K_{2}\left|e_{w, 2}(t)\right|\right) \\
& +\frac{1}{2}\left(K_{3}\left|e_{w, 3}(t)\right|+K_{4}\left|e_{w, 4}(t)\right|\right) \\
= & \frac{1}{2} \sum_{i=1}^{4} K_{i}\left|e_{w, i}(t)\right|
\end{aligned}
$$


In this equation, it is assumed that both "push" muscles ( 1 and 2) had equal relevance, and consequently, the total push force was equal to the mean of both muscles. The same holds for the "pull" muscles ( 3 and 4). Note that $K_{1}$ and $K_{2}$ are positive and $K_{3}$ and $K_{4}$ are negative, as the muscles operate in opposite direction.

2) Nonparametric analysis: The time records $\left(x_{h}(t), f_{h}(t)\right.$, $d(t), a(t))$ of the four repetitions for one condition were averaged to reduce the noise in the signals. The signals were transformed to the frequency domain using the fast Fourier transform (FFT). Because of the interaction between the subject and manipulator (see Fig. 2), closed loop identification algorithms were required to estimate the FRFs of the mechanical admittance and reflexive impedance. The FRFs were estimated by dividing the appropriate spectral densities:

$$
\begin{aligned}
& \hat{H}_{f x}(f)=\frac{\hat{G}_{d x}(f)}{\hat{G}_{d f}(f)} \\
& \hat{H}_{x a}(f)=\frac{\hat{G}_{d a}(f)}{\hat{G}_{d x}(f)}
\end{aligned}
$$

in which $\hat{G}_{d x}$ denotes the estimated cross spectral density between $d$ and $x_{h}$ (hat denotes estimate). $\hat{H}_{f x}$ and $\hat{H}_{x a}$ are estimates for the FRFs of the mechanical admittance and reflexive impedance, respectively. Note that the mechanical admittance describes the mechanical behavior of the joint and incorporated both muscle visco-elasticity and reflexive feedback. The reflexive impedance describes the muscle activation as a result from position deviations and as such is affected by reflexes only. The estimated spectral densities were averaged over four adjacent frequencies to reduce the variance of the estimators [19]. As a measure for reliability, the coherences were calculated

$$
\begin{aligned}
& \hat{\gamma}_{x}^{2}(f)=\frac{\left|\hat{G}_{d x}(f)\right|^{2}}{\hat{G}_{d d}(f) \hat{G}_{x x}(f)} \\
& \hat{\gamma}_{a}^{2}(f)=\frac{\left|\hat{G}_{d a}(f)\right|^{2}}{\hat{G}_{d d}(f) \hat{G}_{a a}(f)} .
\end{aligned}
$$

$\hat{\gamma}_{x}^{2}$ and $\hat{\gamma}_{a}^{2}$ are estimates for the coherence for the handle position and muscle activation, respectively. The coherence varies between 0 and 1 by definition and is reduced by noise and nonlinearities. All estimators for the FRFs and coherences were only evaluated at the frequencies where the force disturbance contained power, unless noted otherwise. The use of periodic multisines as a disturbance ensures that all spectral estimators are unbiased and have relatively low variances [16], [17].

3) Quantitative analysis: To compare the coherences of the muscle activation, the global coherence was calculated for each perturbation. The global coherence, $\bar{\gamma}_{a}{ }^{2}$, is defined as the average of the coherence over the clusters with signal power. Besides the global coherence, the SNR was calculated by dividing the average signal, $S$, by the average noise, $N$, according to

$$
S=\frac{1}{k} \sum \hat{G}_{a a}\left(f_{k}\right)
$$

$$
\begin{aligned}
N & =\frac{1}{n} \sum \hat{G}_{a a}\left(f_{n}\right) \\
\mathrm{SNR} & =10 \log \left(\frac{S}{N}\right)
\end{aligned}
$$

where $f_{k}$ denotes all frequencies between 0.5 and $10 \mathrm{~Hz}$, where the disturbance signal contained power, and $f_{n}$ all frequencies between 0.5 and $10 \mathrm{~Hz}$, where the disturbance signal did not contain power. Note that for the full spectrum condition (WB127), the SNR cannot be obtained because no estimate for the noise can be made. The significance of the effect of the number of clusters in the perturbation signal onto the global coherence and SNR were explored by a two-way analysis of variance (ANOVA).

The coherence and the SNR are highly related. Assume a linear time-invariant system with input $u$ and output $z$, where noise $(n)$ is present in the output

$$
\begin{aligned}
& z=H u+n=y+n \\
& y=H u
\end{aligned}
$$

where $y$ is the part of the output linear related with the input, and $H$ is the linear process between input and output. The theoretical value of the coherence depends on the ratio between the PSDs of $y$ and $n$ [20]

$$
\begin{aligned}
\gamma^{2}(f) & =\frac{\left|G_{u z}(f)\right|^{2}}{G_{u u}(f) G_{z z}(f)} \\
G_{u z}(f) & =H(f) G_{u u}(f) \\
G_{z z}(f) & =|H(f)|^{2} G_{u u}(f)+G_{n n}(f) \\
& =G_{y y}(f)+G_{n n}(f) \\
\gamma^{2}(f) & =\frac{\left|H(f) G_{u u}(f)\right|^{2}}{G_{u u}(f)\left(|H(f)|^{2} G_{u u}(f)+G_{n n}(f)\right)} \\
& =\frac{1}{1+\left(G_{n n}(f) / G_{y y}(f)\right)} .
\end{aligned}
$$

This theoretical value for the coherence shows that coherence is reduced for frequencies with relatively high values of noise.

\section{RESULTS}

\section{A. Isometric Experiments}

Fig. 4 shows the EMG to force relations for a typical subject. The $\mathrm{m}$. pectoralis major and $\mathrm{m}$. deltoideus anterior were active during pushing, while the $\mathrm{m}$. deltoideus posterior and $\mathrm{m}$. latissimus dorsi were relatively silent. On the active part of the force to EMG relation, the EMG to force ratio was estimated with a least square criterion. These ratios are denoted with gray lines in Fig. 4.

\section{B. Main Experiment}

Fig. 5 shows a typical response for reduced spectrum disturbances; left WB64 and right WB22. For both disturbances, the amplitudes in force, position, and activation are comparable. The power of the spectral density of the muscle activation at the 

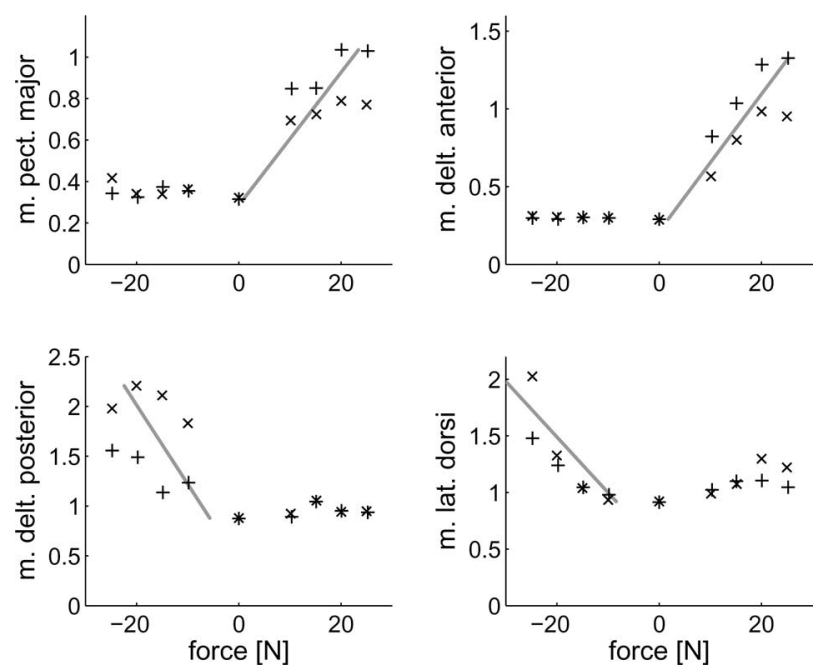

Fig. 4. Force to EMG ratios for a typical subject. Upper-left: m. pectoralis major; upper-right: $\mathrm{m}$. deltoideus anterior; lower-left: $\mathrm{m}$. deltoideus posterior; lower-right: $\mathrm{m}$. latissimus dorsi. Average force is given on the $X$-axis, the corresponding IEMG on the $Y$-axis. Positive force corresponds with pushing. The "x" and "+" denotes the IEMG of the push/pull tasks before and after the main experiment, respectively; the slope of the line denotes the estimated force to EMG ratio.
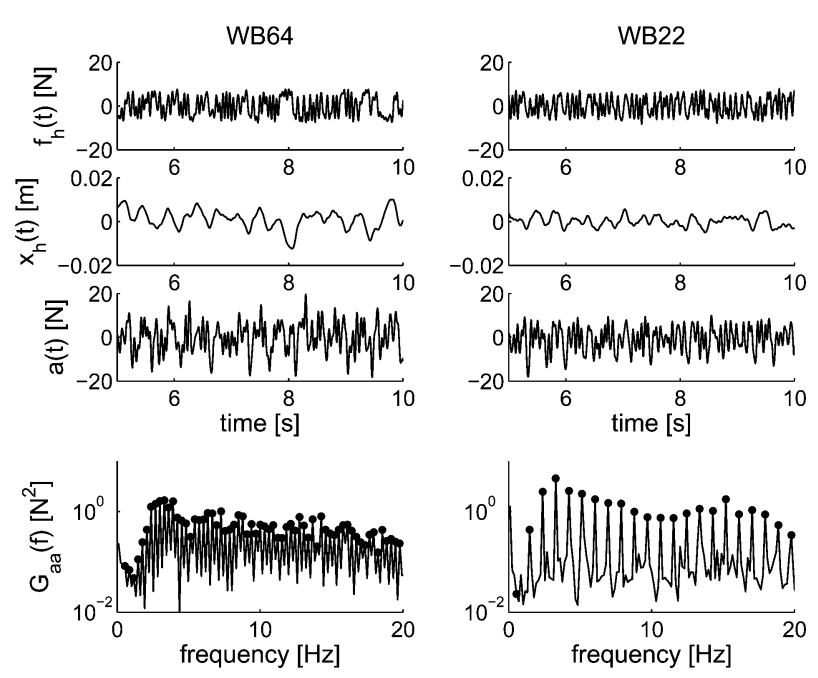

Fig. 5. Time plots of reduced spectrum signals for one subject (left: WB64; right: WB22). First row: force at the handle, $f_{h}(t)$; second row: position deviations, $x_{h}(t)$; third row: muscle activation, $a(t)$. Time plots of muscle activation are filtered (low pass, $20 \mathrm{~Hz}$, third-order Butterworth, forward and backward) for display purposes only. Bottom row: spectral density of the muscle activation, $\hat{G}_{a a}(f)$. Dots denote the frequencies in the disturbance with signal power.

excited frequencies increased when the number of clusters was reduced. This was expected since the input power increased with decreasing number of clusters (see Fig. 3). However, the muscle activation had substantial power in between the clusters, indicating the presence of noise, or nonlinearities. The noise decreased with decreasing number of clusters. Both effects, i.e., increased signal power and decreased noise, contribute to a better SNR with decreasing number of clusters.

Fig. 6 shows the FRFs and coherences for two disturbances for one subject. For clarity, only two of the six conditions are shown, the other four had comparable behavior. The FRF of the
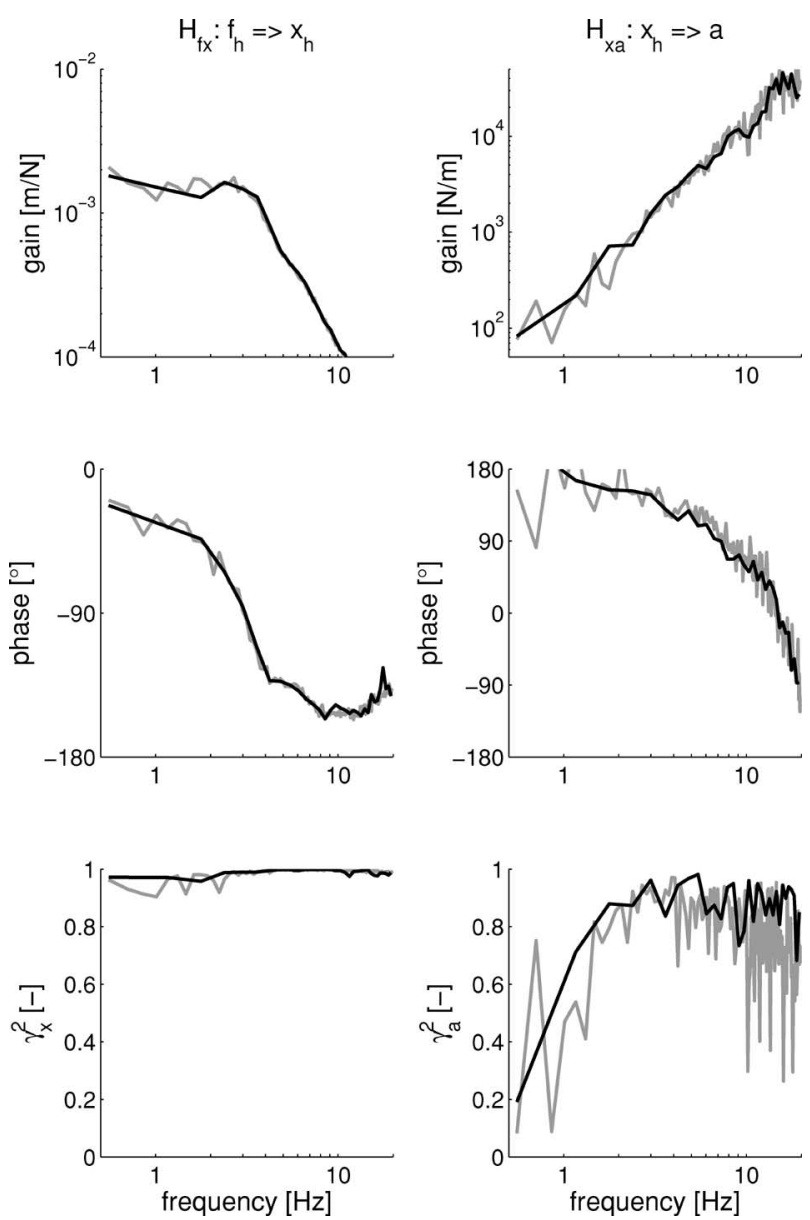

Fig. 6. Gain (upper row) and phase (middle row) of the FRFs for the mechanical admittance (left) and the reflexive impedance (right) for WB127 (gray) and WB32 (black). Bottom row: coherences. Data for one subject are shown.

mechanical admittance (left) did not change with the number of clusters, indicating that the subject responded equally to the disturbances. The coherence for the position (left) was high for all frequencies, indicating that the relation was linear and did not contain substantial noise. The coherence for the muscle activation was relatively low for frequencies below $1 \mathrm{~Hz}$. The gain and phase of the estimated FRF of the reflexive impedance were comparable for all disturbances; however, the variance of the estimator was reduced as the lines become smoother with less clusters.

In the lower plots of Fig. 5, it can be seen that the noise was relatively flat over the frequencies. The signal, however, (denoted with the dots) was substantially lower for the lowest frequencies, and ultimately, had approximately equal power as the noise for the lowest frequencies. As a result, the coherence has a value around 0.5 for the lowest frequencies.

In Table I, the mean EMG, the global coherence, and the SNR for the muscle activation are shown. The mean EMG did not show significant differences between the disturbances, indicating that the muscle cocontraction was constant over the conditions. The global coherence of the muscle activation increased with decreasing number of clusters $(p<0.001)$, as a result of the increased SNR $(p<0.001)$. A disadvantage of 
TABLE I

MeAn EMG, $u_{0}$, Global COHERENCE, ${\overline{\gamma_{a}}}^{2}$, AND THE SNR. MEAN AND STANDARD DEVIATION (SD) OVER All SUbJECTS

\begin{tabular}{lccc}
\hline $\begin{array}{l}\text { Number of } \\
\text { clusters }\end{array}$ & $u_{0}[-]$ & $\bar{\gamma}_{a}{ }^{2}[-]$ & SNR [dB] \\
\hline 127 (=full) & $1(-)$ & $0.63(0.09)$ & $-(-)$ \\
64 & $0.99(0.06)$ & $0.67(0.09)$ & $6.9(1.6)$ \\
43 & $0.99(0.10)$ & $0.69(0.12)$ & $7.6(1.9)$ \\
32 & $0.99(0.10)$ & $0.70(0.14)$ & $9.3(3.4)$ \\
26 & $0.97(0.05)$ & $0.76(0.08)$ & $9.9(1.8)$ \\
22 & $0.99(0.09)$ & $0.75(0.11)$ & $10.6(2.1)$ \\
\hline
\end{tabular}

decreasing the number of clusters was the reduced resolution at which FRFs and coherences could be estimated, as the FRFs could only be evaluated over the frequencies with signal power.

The mechanical admittance (left plots in Fig. 6) resembled a damped second-order system. The stiffness was equal to the inverse of the admittance at $0 \mathrm{~Hz}$ and was, for the arm in the given position, approximately $700 \mathrm{~N} / \mathrm{m}$, and the eigenfrequency was around $3 \mathrm{~Hz}$. From the increasing gain with frequency of the reflexive impedance (upper-right plots in Fig. 6), it appears that the reflexive feedback was dominated by velocity feedback (first derivative of the position, corresponding to a slope of +1 in the gain and $90^{\circ}$ phase lead) and even acceleration feedback (second derivative, slope of $+2,180^{\circ}$ phase lead). The time delay of the proprioceptive reflexes can be derived from the phase of the reflexive impedance. A time delay has unity gain and decreasing phase with increasing frequency. Assuming that the reflexive feedback is only velocity sensitive, the time delay will be around $25 \mathrm{~ms}$. A time delay of $25 \mathrm{~ms}$ would give $180^{\circ}$ phase lag at $20 \mathrm{~Hz}$, the velocity sensitivity would give $90^{\circ}$ phase lead for all frequencies, explaining the observed $90^{\circ}$ phase lag at approximately $20 \mathrm{~Hz}$.

\section{DISCUSSION}

In this paper, a design procedure was presented for the optimization of continuous test signals used for system identification purposes. Here, the procedure was applied to design force disturbance signals for the estimation of linear FRFs of the human neuromuscular system. The procedure adjusted the distribution of signal power over limited frequencies within a prescribed bandwidth of interest. Main criterion was the maximization of the SNR at all frequencies. Optimal test signals enabled the estimation of the dynamic relation between mechanical variables and EMG signals, even though EMG are notorious noisy signals. Satisfactory correlations and coherences were gained which were not possible before. The FRF between hand position and muscle activation was successfully estimated, and this relationship was introduced as the reflexive impedance. The reflexive impedance provides a direct measure of the (lumped) muscle activation that results from reflexive feedback. In this paper, the mechanical admittance [FRF between externally applied force (or reaction force) and hand position] together with the reflexive impedance were estimated using the optimally designed test perturbation signals. Both these relationships provide complementary information of the underlying neuromuscular system of concern that will enable an accurate quantification of neuromuscular properties. Previous studies that aimed to the quantification of spinal reflexes had to rely only on the estimated mechanical admittance, which needed to fix some parameters $a$ priori to avoid parameter redundancy problems. For example, in [7] and [8], joint damping and stiffness from the intrinsic muscles were set to fixed values taken from additional experiments. The same accounted for the neural time delay involved in monosynaptic Ia feedback. The availability of the reflexive impedance in the quantification procedures, such assumptions are not necessary anymore.

A following study will use the current design procedure to accurately quantify proprioceptive reflex gains and neural time delay involved in Ia afferent feedback by fitting a neuromuscular model onto the mechanical admittance and reflexive impedance simultaneously [12]. The effect of disturbance bandwidth and environmental properties on proprioceptive reflexes will be explored.

\section{A. Disturbance Signals}

The disturbances applied contained power between 0.5 and $20 \mathrm{~Hz}$, which was sufficiently wide to capture all relevant neuromechanical control properties of the human shoulder [8]. The signals, applied as force disturbances, were designed in the frequency domain as so-called multisine signals [15], [16]. Multisine signals with random phase give the same expected value for the FRF as Gaussian white noise [21]. Multisines have three important advantages over aperiodic stochastic realizations as also used frequently: 1) no bias is introduced in the spectral estimators; 2) signal properties can be explicitly controlled at specific frequencies; and 3) due to its periodicity, no random errors are introduced. Furthermore, the usage of continuous random force perturbations appeared natural to the subjects and resulted in an unambiguous position holding task.

In the reference test signal, all frequencies within the bandwidth $(0.5-20 \mathrm{~Hz})$ contained power. By distributing the total signal power over fewer frequencies, the power per frequency increased. By application of reduced spectrum signals, the mechanical admittance, the reflexive impedance, and the mean EMG levels of all muscles did not change meaning that the subjects responded in the same way as during application of the reference signal. By distributing the power over fewer frequencies, both the SNR and coherence function between external force and muscle activation increased. Therefore, reducing the number of frequencies is beneficial for studying continuous control behavior. Evidently, FRFs can only be estimated at the frequencies where the test signal contains power. That is, distribution of signal power over a smaller number of frequencies reduces the frequency resolution of the estimated system behavior. Hence, one has to weight the increased SNR against a reduced frequency resolution. Special signals exist to minimize nonlinear distortion [22]. This kind of signals were not used in this study mainly for two reasons: 1) the main interest was the 
linear approximation of the system and not the exact nonlinear distortions and 2) with these signals, the signal power is not equidistantly distributed over the frequencies that could alter the strength of the reflexive feedback [6]-[8].

\section{B. Estimating Muscle Activation from EMG}

In this paper, the neuromuscular behavior of the shoulder was studied. From an additional experiment, static EMG to force relationships of all four muscles were obtained. These EMG signals were taken together to construct one lumped muscle activation signal expressed in newtons. By transforming into newton unity, the EMG signals obtained a direct mechanical meaning and could be used as a variable in a neuromuscular model similar to forces and position.

However, it is questionable if the isometric EMG to force ratios are valid during (small) movements. Only a few studies have mentioned this issue, and in one study, it was found that with equal force, the EMG of the elbow muscles during movements is around $20 \%$ higher, although variations over muscles and subjects were quite large. This difference could only partly be attributed to force-velocity dependency, and the authors found that altered recruitment threshold of the muscles also plays a role [23]. Unfortunately, no data exist on shoulder muscles. If the effect would be present in shoulder muscles, the gain of the reflexive impedance would be slightly overestimated. Furthermore, there is evidence that the gain of the monosynaptic reflex system changes linearly with tonic activation of the homonymous muscle, as measured from the triceps surae and the tibialis anterior [4], [5]. Assuming such a similar relationship for the shoulder muscles as recorded here, the linear EMG to force ratio as obtained during isometric conditions at various tonic contractions seems justified.

Another issue is motor unit synchronicity. The EMG amplitude results from the summation of the muscle's motor unit action potentials. It is known that with transient perturbations, relatively large EMG amplitudes occur as a result of synchronous firing of muscle spindle afferents, and consequently, synchronous motor unit firing. With continuous perturbations, synchronized firing is less likely. The question then remains if synchronous motor unit firing would change the relationship between muscle force and integrated EMG. Since each motor unit contributes to a fixed portion in force and is represented by a fixed part in the EMG signal, the total integrated force and integrated EMG is proportional to the number of motor units, irrespective of the individual timing of firing. The ratio between force and EMG signals is therefore not likely to change with mutual differences in motor unit firing instances.

The assumption that both the muscles that act in the same direction had "equal contribution" need to be stressed a little. Since the muscles recorded here are the major contributors to the forward rotation of the shoulder, one can only state that these muscles do have substantial contribution. The quantitative mutual balance might very well deviate from equality. However, a precise balance is hard to obtain from a noninvasive experimental setting as performed here.

\section{Observed Behavior}

The mechanical admittance (left plots in Fig. 6) resembled a damped second-order system had a stiffness of approximately $700 \mathrm{~N} / \mathrm{m}$ and an eigenfrequency around $3 \mathrm{~Hz}$. Similar values were found in previous studies of the shoulder joint [7], [8]. The reflexive impedance (upper-right plots in Fig. 6) was dominated by a velocity sensitivity in series with a time delay. Many studies under similar conditions of pertubation have shown that reflexive feedback is dominated by a velocity sensitivity as the result of Ia muscle spindle feedback [1], [4]-[6], [24].

The time delay of neural transportation, as captured within the reflexive impedance, was estimated at approximately $25 \mathrm{~ms}$. In many EMG studies, the neural time delay is estimated from the onset of a sudden muscle stretch to the first detectable onset in the EMG signal. However, the current method is based on a frequency-domain analysis using FRFs that are descriptions of correlations between power of signals. This implicates that the estimated delay will be a few milliseconds larger as compared with other studies using onset measures, because the maximum power in the EMG is reached after EMG onset. The exact time lag between onset and maximal power further depends on the location of the EMG electrodes relative to the innervation zone of the efferent motor fibers and on the inter electrode distance.

\section{REFERENCES}

[1] K. Akazawa, T. E. Milner, and R. B. Stein, "Modulation of reflex emg and stiffness in response to stretch of human finger muscle," J. Neurophysiol., vol. 49, pp. 16-27, 1983.

[2] F. Doemges and P. M. H. Rack, "Task-dependent changes in the response of human wrist joints to mechanical disturbance," J. Physiol.-London, vol. 447, pp. 575-585, 1992.

[3] R. B. Stein and C. Capaday, "The modulation of human reflexes during functional motor-tasks," Trends Neurosci., vol. 11, pp. 328-332, 1988.

[4] R. E. Kearney and I. W. Hunter, "System identification of human triceps surae stretch reflex dynamics," Exp. Brain Res., vol. 51, pp. 117-127, 1983.

[5] R. E. Kearney and I. W. Hunter, "System identification of human stretch reflex dynamics: Tibialis anterior,” Exp. Brain Res., vol. 56, pp. 40-49, 1984.

[6] R. E. Kearney, R. B. Stein, and L. Parameswaran, "Identification Of intrinsic and reflex contributions to human ankle stiffness dynamics," IEEE Trans. Biomed. Eng., vol. 44, pp. 493-504, 1997.

[7] E. de Vlugt, A. C. Schouten, and F. C. T. van der Helm, "Adaptation of reflexive feedback during arm posture to different environments," Biol. Cybern., vol. 87, pp. 10-26, 2002.

[8] F. C. T. van der Helm, A. C. Schouten, E. de Vlugt, and G. G. Brouwn, "Identification of intrinsic and reflexive components of human arm dynamics during postural control," J. Neurosci. Methods, vol. 119, pp. 1-14, 2002.

[9] A. C. Schouten, W. J. T. Van de Beek, J. J. Van Hilten, and F. C. T. Van der Helm, "Proprioceptive reflexes in patients with reflex sympathetic dystrophy," Exp. Brain Res., vol. 151, pp. 1-8, 2003.

[10] A. C. Schouten, E. de Vlugt, F. C. T. Van der Helm, and G. G. Brouwn, "Optimal posture control of a musculo-skeletal arm model," Biol. Cybern., vol. 84, pp. 143-152, 2001.

[11] E. de Vlugt, F. C. T. Van der Helm, A. C. Schouten, and G. G. Brouwn, "Analysis of the reflexive feedback control loop during posture maintenance," Biol. Cybern., vol. 84, pp. 133-141, 2001.

[12] A. Schouten, E. de Vlugt, J. van Hilten, and F. van der Helm, "Quantifying proprioceptive reflexes during position control of the human arm," IEEE Trans. Bio-Med. Eng., vol. 55, no. 1, pp. 311-321, Jan. 2008.

[13] J. Ruitenbeek and R. Janssen, "Computer-controlled manipulator/display system for human-movement studies," Med. Biol. Eng. Comput., vol. 22, pp. 304-308, 1984.

[14] G. Wu, F. van der Helm, H. Veeger, M. Makhsouse, P. Van Roy, C. Angling, J. Nagels, A. Kardunai, K. McQuade, X. Wang, F. Werner, 
and B. Buchholz, "Isb recommendation on definitions of joint coordinate systems of various joints for the reporting of human joint motion-Part II: Shoulder, elbow, wrist and hand," J. Biomech., vol. 38, pp. 981-992, 2005.

[15] J. Schoukens, P. Guillaume, and R. Pintelon, Design of Broadband Excitation Signals, K. Godfrey, Ed. Englewood Cliffs, NJ: Prentice-Hall, 1993, 2008.

[16] R. Pintelon and J. Schoukens, System Identification: A Frequency Domain Approach. New York: IEEE Press, 2001.

[17] E. de Vlugt, A. C. Schouten, and F. C. T. van der Helm, "Closed-loop multivariable system identification for the characterization of the dynamic arm compliance using continuous force disturbances: a model study," $J$. Neurosci. Methods, vol. 122, pp. 123-140, 2003.

[18] E. A. Clancy, E. L. Morin, and R. Merletti, "Sampling, noise-reduction and amplitude estimation issues in surface electromyography," J. Electromyography Kinesiology, vol. 12, pp. 1-16, 2002.

[19] G. Jenkins and D. Watts, Spectral Analysis and Its Applications. San Francisco, CA: Holden-Day, 1968.

[20] D. Westwick and R. Kearney, Identification of Nonlinear Physiological Systems. New York: IEEE Press, 2003.

[21] J. Schoukens, T. Dobrowiecki, and R. Pintelon, "Parametric and nonparametric identification of linear systems in the presence of nonlinear distortions-A frequency domain approach," IEEE Trans. Automat. Control, vol. 43, no. 2, pp. 176-190, Feb. 1998.

[22] B. Suki and K. R. Lutchen, "Pseudorandom signals to estimate apparent transfer and coherence functions of nonlinear-systems-Applications to respiratory mechanics," IEEE Trans. Biomed. Eng., vol. 39, pp. 11421151, 1992.

[23] M. Theeuwen, C. Gielen, and L. Miller, "The relative activation of muscles during isometric contractions and low-velocity movements against a load," Exp. Brain Res., vol. 101, pp. 493-505, 1994.

[24] M. M. Mirbagheri, H. Barbeau, and R. E. Kearney, "Intrinsic and reflex contributions to human ankle stiffness: Variation with activation level and position," Exp. Brain Res., vol. 135, pp. 423-436, 2000.

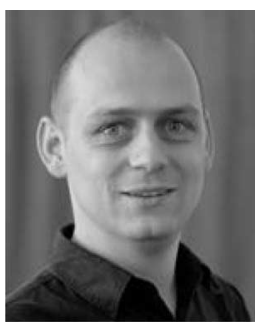

Alfred C. Schouten received the M.Sc. and Ph.D. degrees in mechanical engineering from Delft University of Technology, Delft, The Netherlands, in 1999 and 2004, respectively.

$\mathrm{He}$ is currently an Assistant Professor at the Department of Biomechanical Engineering, Delft University of Technology. He is the Co-Founder of the Delft Laboratory for Neuromuscular Control. His current research interests include neuromuscular control and includes techniques to quantify the functional contribution of afferent feedback, neuromuscular modelling, haptic manipulators, and system identification.

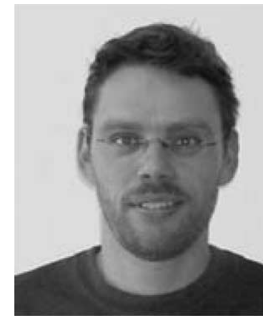

Erwin de Vlugt received the M.Sc. and Ph.D. degrees in mechanical engineering from Delft University of Technology (DUT), Delft, The Netherlands, in 1998 and 2004, respectively.

$\mathrm{He}$ is currently an Assistant Professor at the Biomechanical Engineering Department, DUT. He is the Co-Founder of Delft Laboratory for Neuromuscular Control (NMC Lab). His current research interests include modulation of spinal reflexes in vivo, both in the normal and pathological cases, system identification, neuromuscular modeling, and haptic robotics. Other research activities are dedicated to system identification, neuromuscular modeling and haptic robotics.

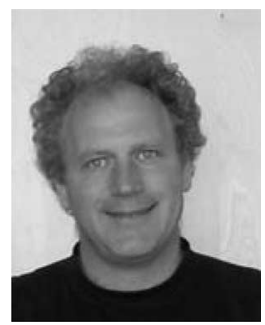

Frans C. T. van der Helm received the M.Sc. degree in human movement science from VU University Amsterdam, The Netherlands, in 1985 and the Ph.D. degree in mechanical engineering from Delft University of Technology, Delft, The Netherlands, in 1991.

$\mathrm{He}$ is currently the Professor in Biomechatronics and Biorobotics and the Head of the Department of Biomechanical Engineering Delft University of Technology, Delft, The Netherlands. He is one of the program leaders in the Delft Center on Biomedical Engineering. He is Principal Investigator in the Trauma Related Neuronal Dysfunction (TREND) research consortium, investigating Complex Regional Pain Syndrome as a neurological disorder. He is the author or coauthor of more than 100 papers in international journals. His current research interests include biomechanics of the upper and lower extremity, neuromuscular control, eye biomechanics, pelvic floor biomechanics, human motion control, and posture stability.

Dr. Helm is a member of the board of the International Society of Biomechanics (ISB), and participates in the board of the Technical Group of Computer Simulation (TGCS) and the International Shoulder Group (ISG). 